$\mathrm{UCLA} / 99 / \mathrm{TEP} / 15$

MPI-PhT/99-17

April 1999

\title{
Prompt atmospheric neutrinos and muons: NLO vs LO QCD predictions
}

\author{
Graciela Gelmini, ${ }^{1}$ Paolo Gondolo, ${ }^{2}$ Gabriele Varieschi ${ }^{1}$ \\ ${ }^{1}$ Dept. of Physics and Astronomy, UCLA (University of California, Los Angeles) \\ 405 Hilgard Ave., Los Angeles CA 90095, USA \\ gelmini,variesch@physics.ucla.edu \\ ${ }^{2}$ Max-Planck-Institut für Physik (Werner-Heisenberg-Institut) \\ Föhringer Ring 6, 80805 München, Germany \\ gondolo@mppmu.mpg.de
}

\begin{abstract}
We compare the leading and next-to-leading order QCD predictions for the flux of atmospheric muons and neutrinos from decays of charmed particles. We find that the full NLO lepton fluxes can be approximated to within $\sim 10 \%$ by the Born-level fluxes multiplied by an overall factor of $2.2-2.4$, which depends slightly on the PDF. This supports the approach in Thunman, Ingelman, Gondolo (1996). We also find that their very low lepton fluxes are due to the mild slope they used for the gluon distribution function at small momentum fractions, and that substantially larger lepton fluxes result when the slope of the gluon distribution function at small momentum fractions is larger.
\end{abstract}




\section{Introduction}

The flux of atmospheric neutrinos and muons at very high energies, above $1 \mathrm{TeV}$, passes from being originated in the decays of pions and kaons to being predominantly generated in semileptonic decays of charmed particles (see for example [1]). This flux is of importance for large area detectors of high energy cosmic neutrinos. Future $\mathrm{km}^{3}$ arrays would be able to observe muons and neutrinos with energies that may reach $10^{12}$ $\mathrm{GeV}$. Atmospheric muons and neutrinos would be one of the most important backgrounds, limiting the sensitivity of any "neutrino telescope" to astrophysical signals. Besides, they might be used for detector calibration and perhaps, more interestingly, be exploited to do physics, e.g. study neutrino masses.

Present experimental attempts to detect atmospheric muons from charm are spoiled by systematic errors. Theoretical predictions depend strongly on the reliability of the model adopted for charm production and decay and differ by orders of magnitude, due to the necessity of extrapolating present accelerator data on open charm production in fixed target experiments, at laboratory energies of about $200 \mathrm{GeV}$, to the larger energies needed for atmospheric neutrinos, from $10^{3}$ to $10^{8} \mathrm{GeV}$ (at about $10^{8} \mathrm{GeV}$ the rates become too small for a $\mathrm{km}^{3}$ detector). These energies, from $40 \mathrm{GeV}$ to $14 \mathrm{TeV}$ in the center of mass, are comparable to the energies of the future RHIC at Brookhaven, $200 \mathrm{GeV}$, and LHC at CERN, $7 \mathrm{TeV}$.

The theoretically preferred model, perturbative QCD (pQCD), was thought to be inadequate because it could not account for several aspects of some of the early data on open charm production (in conflict with each other, on the other hand [2]), and because of a sensitivity of the leading-order (LO) calculation, the only existing until recently, to the charm quark mass, to the low partonic momentum fraction, $x$, behavior of the parton distributions and to higher order corrections. So, even if some nowobsolete pQCD calculations have appeared [3, 4, the models for charm production traditionally favored in studies of atmospheric fluxes have been non-perturbative: for example, besides semi-empirical parametrizations of the cross section, the quark-gluon string model (QGSM, a.k.a. dual parton model), based on Regge asymptotics, and the recombination quark-parton model (RQPM), incorporating the assumption of an intrinsic charm component in the nucleon (see [5]).

Today, however, pQCD predictions and experimental data are known to be compatible [6, 7, 8, 9, 10]: charm production experiments form a consistent set of data, and the inclusion of next-to-leading order (NLO) terms has been a major improvement over the leading-order treatment. Quoting from Appel [6], "the success of these calculations has removed the impetus to look for unconventional sources of charm production beyond the basic QCD".

A study based on pQCD was therefore performed in Ref. [11] (called TIG from now on). CLEO and HERA results were incorporated, but for simplicity the LO charm production cross section was adopted, multiplied by a constant $K$ factor of 2 to bring it in line with the next-to-leading order values, and supplemented by parton shower evolution and hadronization according to the Lund model. The neutrino and 
muon fluxes from charm were found to be lower than the lowest previous prediction, namely a factor of 20 below the RQPM [12], of 5 below the QGSM [13, 14], and of 3 below the lowest curve in Ref. [4].

Here we use the same treatment of TIG, except for the very important difference of using the actual next-to-leading order pQCD calculations of Mangano, Nason and Ridolfi [15] (called MNR from now on), as contained in the program we obtained from them (see also [16]), to compute the charm production cross sections. These are the same calculations used currently to compare pQCD predictions with experimental data in accelerator experiments. The main goal of this paper is to compare the fluxes obtained with the NLO and with the LO, i.e. we will compute the $K$ factor for the neutrino and muon fluxes. This $K$ factor is necessarily different from the $K$ factor for charm production (which can be found in the literature), because only the forward going leptons contribute significantly to the atmospheric fluxes.

A similar comparison was very recently made in [17], using the approximate analytical solutions introduced by TIG to the cascade equations in the atmosphere. We make instead a full simulation of the cascades, using the combined MNR and PYTHIA programs. These two treatments of the problem are complementary. For comparison, we include results obtained with the CTEQ 3M gluon structure function used in Ref. [17]. We find our CTEQ 3M results to be close to those of the PRS study, in spite of the very different approaches used in the two calculations.

Addressing right away a concern that has been expressed to us several times, about the applicability of perturbative QCD calculations, mostly done for accelerator physics, to the different kinematic domain of cosmic rays, we would like to point out that, since the characteristic charm momentum in our simulations is of the order of the charm mass, $k \simeq O\left(m_{c}\right)$, we do not have here the uncertainty present in the differential cross sections [15], when $k_{T}$ is much larger than $m_{c}$ (as is the case in accelerators), due to the presence of large logarithms of $\left(k_{T}^{2}+m_{c}^{2}\right) / m_{c}^{2}$. Depending on the steepness of the gluon structure function we take, we do have, however, large logarithms, known as " $\ln (1 / \mathrm{x})$ " terms, where $x \simeq \sqrt{4 m_{c}^{2} / s}$ ( $s$ is the hadronic center of mass energy squared $)$ is the average value of the hadron energy fraction needed to produce the $c \bar{c}$ pair. These should not be important for steep enough gluon structure functions (namely for values of $\lambda$ in Eq. (9) not very close to zero), but we have not made any attempt to deal with this issue.

In the next section of this paper we explain our normalization of the NLO charm production cross section in the MNR program. In Sect. 3 we describe the computer simulations used to calculate the neutrino and muon fluxes. In Sect. 1 we show the results of our simulations, we discuss the differences between a NLO and a LO approach and we make a comparison with the fluxes of the TIG model.

In this paper we consider only vertical showers for simplicity (the same was done by TIG). 


\section{Charm production in perturbative QCD}

In this section, we show evidence that perturbative QCD gives a fair description of the present accelerator data on open charm production in the kinematic region most important for cosmic ray collisions in the atmosphere.

There are still not many experiments on open charm production with good enough statistics, despite the recent improvements, but many are expected in the near future.

We use a NLO approach which is based on the MNR calculation, for which we have obtained the computer code. The NLO cross section for charm production depends on the choice of the parton distribution functions (PDFs) and on three parameters: the charm quark mass $m_{c}$, the renormalization scale $\mu_{R}$, and the factorization scale $\mu_{F}$.

\subsection{Choice of $m_{c}, \mu_{R}, \mu_{F}$}

MNR have two default choices of $m_{c}, \mu_{R}$ and $\mu_{F}$ : for total cross sections they choose $m_{c}=1.5 \mathrm{GeV}, \mu_{R}=m_{c}, \mu_{F}=2 m_{c}$; for differential cross sections they choose instead $m_{c}=1.5 \mathrm{GeV}, \mu_{R}=m_{T}, \mu_{F}=2 m_{T}$, where $m_{T}=\sqrt{k_{T}^{2}+m_{c}^{2}}$ is the transverse mass.

The current procedure to reproduce the measured differential cross sections [8, 9, 10] is to use the MNR default choices for these three parameters and multiply the result by the global factor of about 2 or 3 necessary to match the predicted and measured total inclusive cross sections. Although this procedure might be acceptable in face of the uncertainties in the pQCD predictions, we find it unsatisfactory from a theoretical point of view. We prefer to fit the differential and total cross sections with one and the same combination of $m_{c}, \mu_{R}$, and $\mu_{F}$.

We make separate fits of $m_{c}, \mu_{R}$, and $\mu_{F}$ for each of the following sets of PDFs: MRS R1, MRS R2 [18], CTEQ 3M [19] and CTEQ 4M 20] (see the next subsection for details).

We are aware that several choices of $m_{c}, \mu_{R}$ and $\mu_{F}$ may work equally well. In fact the cross sections increase by decreasing $\mu_{F}, \mu_{R}$ or $m_{c}$, so changes in the three variables can be played against each other to obtain practically the same results. We present here just one such choice.

We choose $\mu_{R}=m_{T}, \mu_{F}=2 m_{T}$ for all sets, and

$$
\begin{array}{ll}
m_{c}=1.185 \mathrm{GeV} & \text { for MRS R1, } \\
m_{c}=1.31 \mathrm{GeV} & \text { for MRS R2, } \\
m_{c}=1.24 \mathrm{GeV} & \text { for CTEQ 3M, } \\
m_{c}=1.27 \mathrm{GeV} & \text { for CTEQ } 4 \mathrm{M} .
\end{array}
$$

We fit $m_{c}, \mu_{R}$, and $\mu_{F}$ to the latest available data on charm production [7, 8, 9, 10, in proton-nucleon and pion-nucleon collisions. We use mainly the data on $p N$ collisions, which are more relevant to us, but examine also the $\pi N$ data to see how well our choice of parameters works there.

The MNR program calculates the total cross section for $c \bar{c}$ pair production, $\sigma_{c \bar{c}}$. We converted the experimental data on $D^{+}$or $D^{-}$production $\sigma\left(D^{+}, D^{-}\right), D^{0}$ or $\bar{D}^{0}$ 
production $\sigma\left(D^{0}, \bar{D}^{0}\right)$, or the same cross sections just for $x_{F}>0\left(x_{F}\right.$ is the Feynman $x), \sigma_{+}\left(D^{+}, D^{-}\right)$and $\sigma_{+}\left(D^{0}, \bar{D}^{0}\right)$, into $\sigma_{c \bar{c}}$ values following [10].

The data we used for the 'calibration' of the MNR program are shown in Table 1 and Table 2 [7, 8, 9, 10]. These tables also present a comparison of experimental data on total inclusive D-production cross sections (converted to $\sigma_{c \bar{c}}$ total cross sections) with those calculated with the MNR program.

For the data of Table 1, for $p N$ collisions, the conversion is done using

$$
\sigma_{c \bar{c}}=1.5 \times \frac{1}{2} \times\left[\sigma\left(D^{+}, D^{-}\right)+\sigma\left(D^{0}, \bar{D}^{0}\right)\right]
$$

if cross sections are measured for any $x_{F}$, or

$$
\sigma_{c \bar{c}}=1.5 \times 2 \times \frac{1}{2}\left[\sigma_{+}\left(D^{+}, D^{-}\right)+\sigma_{+}\left(D^{0}, \bar{D}^{0}\right)\right]
$$

if experimental data are given for $x_{F}>0$ only. The explanation of the factors in Eqs. (5),(6) is as follows. The $\frac{1}{2}$ factors convert single $D$ inclusive into $D \bar{D}$ pair inclusive cross sections. The 1.5 factors are required to take into account the production of $D_{S}$ and $\Lambda_{c}$ (which is included in $\sigma_{c \bar{c}}$ ) through the ratios [10]

$$
\frac{\sigma\left(D_{S}\right)}{\sigma\left(D^{+}, D^{0}\right)} \simeq 0.2, \quad \frac{\sigma\left(\Lambda_{c}\right)}{\sigma\left(D^{+}, D^{0}\right)} \simeq 0.3
$$

(the same relation also for antiparticles). The factor 2 in Eq. (6) converts from $x_{F}>0$ to all $x_{F}$ (i.e. it is $\sigma_{c \bar{c}} / \sigma_{c \bar{c}}\left(x_{F}>0\right)$ for the $p N$ case).

In the case of $\pi N$ collisions (Table 2) the factor 2 in equation (6) is replaced by 1.6, which is the value of $\sigma_{c \bar{c}} / \sigma_{c \bar{c}}\left(x_{F}>0\right)$ when a pion beam is used.

Table 11 explains our choice of $m_{c}$ values. The $m_{c}$ values in Eqs.(11),(2),(3) and (4) reproduce well the central values of the $p N$ charm inclusive total cross sections [7], using the program with the four different PDFs.

In Table 2 we also present a similar analysis for $\pi N$ collisions, using only MRS $\mathrm{R} 1$ for simplicity. In this case slightly higher values of $m_{c}$ fit the $\pi N$ data [7, 10] a bit better, while $m_{c}=1.185 \mathrm{GeV}$, the value we take with the MRS R1 PDF, fits the $p N$ data [0, 8, 10] a bit better. Notice that for the pions we used a different PDF, SMR2 [21], the same used in Refs. [7, 8] (obviously not used in our calculations of atmospheric fluxes). We present the $\pi N$ data just for completeness, to show that they too are reasonably well fitted with our choice of parameters. These other values of $m_{c}$ in Table 2 2 well reproduce the $\pi^{ \pm} N$ data at $250 \mathrm{GeV}$ [7] and the $\pi^{-} N$ data at $350 \mathrm{GeV}$ [9] (which seem a bit too low with respect to the data at $250 \mathrm{GeV}$ ). Even if each value of $m_{c}$ reproduces best each total cross section, all three provide reasonable fits to all data, as can be seen also in the Figs. 1-3.

In Figs. 1-3 we present total and differential cross sections calculated with the MNR program and compared to the experimental data. As a way of example, we describe our fits for MRS R1 only.

Fig. 1a shows the fit to $p N$ total cross sections (converted into $\sigma_{c \bar{c}}$ values as described above). In addition to the experimental value of Table 1 - which is the fundamental 
one, since it's the experiment whose differential cross sections we want also to fit we added other experimental points coming from previous experiments (for details see [10]). For $p N$ the $m_{c}=1.185 \mathrm{GeV}$ is the best choice.

Fig. 1b shows the same for $\pi N$ collisions. Here, as explained before, values of $m_{c}=1.25 \mathrm{GeV}$ or $m_{c}=1.31 \mathrm{GeV}$ are a better choice. Again we added here for completeness other experimental points coming from previous experiments [10].

Fig. 2ab shows fits to D-inclusive differential cross sections. In this figure the theoretically obtained $d \sigma_{c \bar{c}} / d x_{F}$ and $d \sigma_{c \bar{c}} / d p_{T}^{2}$ were converted into D-cross sections, with no extra factors. Fig. 2ab presents the data of the E769 collaboration [8] for $p N$ and $\pi N$ at $250 \mathrm{GeV}$. In these cases the differential $\sigma_{c \bar{c}}$ cross sections are converted into single inclusive ones (by a factor of 2) and then into cross sections for production of $D^{ \pm}, D^{0}, \bar{D}^{0}$ and $D_{S}^{ \pm}$(by a factor of $1.2 / 1.5$, see Eq. (7) ) for the E769 data. For example,

$$
\frac{d \sigma}{d x_{F}}\left(D^{ \pm}, D^{0}, \bar{D}^{0}, D_{S}^{ \pm}\right) \simeq \frac{1.2}{1.5} \times 2 \times \frac{d \sigma_{c \bar{c}}}{d x_{F}}
$$

for Fig. 2a (and similar factors for $d \sigma / d p_{T}^{2}$ for Fig. 2b). The fit to the $d \sigma / d p_{T}^{2} \quad p N$ data in Fig. 2b seems to be a bit too low, but it is not very different from the fit shown in Fig. 2 of reference [8]. The predicted $d \sigma / d p_{T}^{2}$ are not sensitive to differences in $m_{c}$ that are instead more noticeable in $d \sigma / d x_{F}$.

Fig. 3ab presents the $\pi N$ data at $350 \mathrm{GeV}$ of the WA92 collaboration [9] in a way similar to Fig. 2ab. In these cases the differential $\sigma_{c \bar{c}}$ cross sections are converted into a single inclusive ones (by a factor of 2 ) and then into cross sections for production of $D^{ \pm}, D^{0}$ and $\bar{D}^{0}$ only (by a factor of $1.0 / 1.5$, see Eq. (])) for the WA92 data. Similar conclusions can be drawn: for pions $m_{c}=1.31 \mathrm{GeV}$ is the best choice in this case.

We have performed the same analysis with MRS R2, CTEQ 4M and CTEQ 3M, even if we do not show here any of the fits. The results for total and differential cross sections were similar to those shown for the MRS R1, the only difference being the choice of $m_{c}$.

In conclusion, we obtain good fits to all data on charm production with one choice of $\mu_{R}, \mu_{F}$ and $m_{c}$ for each PDF, without other normalizations.

\subsection{Choice of PDFs}

Consider the collision of a cosmic ray nucleus of energy $E$ per nucleon, with a nucleus of the atmosphere in which charm quarks of energy $E_{c}$ are produced, which decay into leptons of energy $E_{l}$ (in the lab. frame, namely the atmosphere rest frame). Due to the steep decrease with increasing energy of the incoming flux of cosmic rays, only the most energetic charm quarks produced count for the final lepton flux, and these $c$ quarks come from the interactions of projectile partons carrying a large fraction of the incoming nucleon momentum. Thus, the characteristic $x$ of the projectile parton, that we call $x_{1}$, is large. It is $x_{1} \simeq O\left(10^{-1}\right)$. We can, then, immediately understand that very small parton momentum fractions are needed in our calculation, because typical 
partonic center of mass energies $\sqrt{\hat{s}}$ are close to the $c \bar{c}$ threshold, $2 m_{c} \simeq 2 \mathrm{GeV}$ (since the differential cross section decreases with increasing $\hat{s}$ ), while the total center of mass energy squared is $s=2 m_{N} E$ (with $m_{N}$ the nucleon mass, $m_{N} \simeq 1 \mathrm{GeV}$ ). Calling $x_{2}$ the momentum fraction of the target parton (in the nuclei of the atmosphere), then, $x_{1} x_{2} \equiv \hat{s} / s=4 m_{c}^{2} /\left(2 m_{N} E\right) \simeq \mathrm{GeV} / E$. Thus, $x_{2} \simeq O(\mathrm{GeV} / 0.1 E)$, where $E$ is the energy per nucleon of the incoming cosmic ray in the lab. frame. The characteristic energy $E_{c}$ of the charm quark and the dominant leptonic energy $E_{l}$ in the fluxes are $E_{l} \simeq E_{c} \simeq 0.1 E$, thus $x_{2} \simeq O\left(\mathrm{GeV} / E_{l}\right)$, as mentioned above.

For $x>10^{-5}\left(E \lesssim 10^{3} \mathrm{TeV}\right)$, PDFs are available from global analyses of existing data. We use four sets of PDFs. MRS R1, MRS R2 [18] and CTEQ 4M [20, incorporate most of the latest HERA data and cover the range of parton momentum fractions $x \geq 10^{-5}$ and momentum transfers $Q^{2} \geq 1.25-2.56 \mathrm{GeV}^{2}$. MRS R1 and MRS R2 differ only in the value of the strong coupling constant $\alpha_{s}$ at the $\mathrm{Z}$ boson mass: in MRS $\mathrm{R} 1 \alpha_{s}\left(M_{Z}^{2}\right)=0.113$, and in MRS R2 $\alpha_{s}\left(M_{Z}^{2}\right)=0.120$. The former value is suggested by "deep inelastic scattering" experiments, and the latter by LEP measurements. This difference leads to different values of the PDF parameters at the reference momentum $Q_{0}^{2}=1.25 \mathrm{GeV}^{2}$ where the QCD evolution of the MRS R1 and R2 PDFs is started. The CTEQ $4 \mathrm{M}$ is the standard choice in the $\overline{M S}$ scheme in the most recent group of PDFs from the CTEQ group $\left(\alpha_{s}\left(M_{Z}^{2}\right)=0.116\right.$ for CTEQ $\left.4 \mathrm{M}\right)$. We also use an older PDF by the CTEQ group, namely the CTEQ 3M [19], only for comparisons with [17], where it is used as the main PDF.

For $x<10^{-5}\left(E \gtrsim 10^{3} \mathrm{TeV}\right)$, we need to extrapolate the available PDFs. For $x \ll 1$, all these PDFs go as

$$
x f_{i}\left(x, Q^{2}\right) \simeq A_{i} x^{-\lambda_{i}\left(Q^{2}\right)},
$$

where $i$ denotes valence quarks $u_{v}, d_{v}$, sea quarks $S$, or gluons $g$. The PDFs we used (except the older CTEQ 3M) have $\lambda_{S}\left(Q_{0}^{2}\right) \neq \lambda_{g}\left(Q_{0}^{2}\right)$, in contrast to older sets of PDFs which assumed an equality. As $x$ decreases the density of gluons grows rapidly. At $x \simeq 0.3$ it is comparable to the quark densities but, as $x$ decreases it increasingly dominates over the quark densities, which become negligible at $x \lesssim 10^{-3}$.

We need, therefore, to extrapolate the gluon PDFs to $x<10^{-5}$. Extrapolations based on Regge analysis usually propose $x g(x) \sim x^{-\lambda}$ with $\lambda \simeq 0.08$ [22], while evolution equations used to resum the large logarithms $\alpha_{s} \ln (1 / x)$ mentioned above, such as the BFKL (Balitsky, Fadin, Kuraev, Lipatov [23]) find also $x g(x) \sim x^{-\lambda}$ but with $\lambda \simeq 0.5$ [22]. A detailed analysis of the dependence of the neutrino fluxes on the low $x$ behavior of the PDFs will be given in another publication [24]. As mentioned above, in the present paper our goal is to compare NLO to BORN simulations, for which we use a simplified extrapolation at low $x$ of the gluon PDF, which is somewhat in between the two extreme theoretical behaviors described above. For MRS R1-R2 and CTEQ 4M we take a linear extrapolation of $\ln g(x)$ as a function of $\ln x$, in which we took $\ln g(x)=-\left(\lambda_{g}\left(Q^{2}\right)+1\right) \ln x+\ln A_{g}$, where $\lambda_{g}\left(Q^{2}\right)$ was taken as its value at $x=10^{-5}$, the smallest $x$ for which the PDFs are provided; for the CTEQ 3M we used a polynomial approximation which is included in the PDF package. 


\section{Simulation of particle cascades in the atmosphere}

We simulate the charm production process in the atmosphere and the subsequent particle cascades, by modifying and combining together two different programs: the MNR routines [15] and PYTHIA 6.115 [25].

The MNR program was modified to become an event generator for charm production at different heights in the atmosphere and for different energies of the incoming primary cosmic rays.

The charm quarks (and antiquarks) generated by this first stage of the program are then fed into a second part which handles quark showering, fragmentation and the interactions and decays of the particles down to the final leptons. The cascade evolution is therefore followed throughout the atmosphere: the muon and neutrino fluxes at sea level are the final output of the process.

In this section we give a brief description of the main parts of the simulation. Even if our program is completely different from the one used by TIG, because it is constructed around the MNR main routines, nevertheless we keep the same modeling of the atmosphere and of the primary cosmic ray flux as in TIG and the same treatment of particle interactions and decays in the cascade.

Our main improvement is the inclusion of a true NLO contribution for charm production (and updated PDFs), so we keep all other assumptions of the TIG model in order to make our results comparable to those of TIG. We study the effect of modifying some of their other assumptions elsewhere 24].

\subsection{The model for the atmosphere}

We assume a simple isothermal model for the atmosphere. Its density at vertical height $h$ is

$$
\rho(h)=\frac{X_{0}}{h_{0}} e^{-h / h_{0}},
$$

where the scale height $h_{0}=6.4 \mathrm{~km}$ and the column density $X_{0}=1300 \mathrm{~g} / \mathrm{cm}^{2}$ at $h=0$ are chosen as in TIG, to fit the actual density in the range $3 \mathrm{~km}<h<40 \mathrm{~km}$, important for cosmic ray interactions. Along the vertical direction, the amount of atmosphere traversed by a particle, the depth $X$, is related to the height $h$ simply by

$$
X=\int_{h}^{\infty} \rho\left(h^{\prime}\right) d h^{\prime}=X_{0} e^{-h / h_{0}} .
$$

The atmospheric composition at the important heights is approximately constant: $78.4 \%$ nitrogen, $21.1 \%$ oxygen and $0.5 \%$ argon with average atomic number $\langle A\rangle=$ 14.5 . 


\subsection{The primary cosmic ray flux}

Following TIG [1]], we neglect the detailed cosmic ray composition and consider all primaries to be nucleons with energy spectrum

$$
\phi_{N}(E, 0)\left[\frac{\text { nucleons }}{\mathrm{cm}^{2} \mathrm{~s} \mathrm{sr} \mathrm{GeV} / \mathrm{A}}\right]= \begin{cases}1.7(E / \mathrm{GeV})^{-2.7} & \text { for } E<510^{6} \mathrm{GeV} \\ 174(E / \mathrm{GeV})^{-3} & \text { for } E>510^{6} \mathrm{GeV}\end{cases}
$$

The primary flux is attenuated as it penetrates into the atmosphere by collisions against the air nuclei. An approximate expression for the intensity of the primary flux at a depth $X$ is (see [11] again)

$$
\phi_{N}(E, X)=e^{-X / \Lambda_{N}(E)} \phi_{N}(E, 0) .
$$

The nuclear attenuation length $\Lambda_{N}$, defined as

$$
\Lambda_{N}(E)=\frac{\lambda_{N}(E)}{1-Z_{N N}(E)}
$$

has a mild energy dependence through $Z_{N N}$ and $\lambda_{N}$. Here $Z_{N N}$ is the spectrumweighted moment for nucleon regeneration in nucleon-nucleon collisions, for which we use the values in Fig. 4 of Ref. [11]. And $\lambda_{N}$ is the interaction thickness

$$
\lambda_{N}(E, h)=\frac{\rho(h)}{\sum_{A} \sigma_{N A}(E) n_{A}(h)},
$$

where $n_{A}(h)$ is the number density of air nuclei of atomic weight $A$ at height $h$ and $\sigma_{N A}(E)$ is the total inelastic cross section for collisions of a nucleon $N$ with a nucleus A.f This cross section scales essentially as $A^{2 / 3}$, since for the large nucleon-nucleon cross sections we deal with, the projectiles do not penetrate the nucleus. So we set $\sigma_{N A}(E)=A^{2 / 3} \sigma_{N N}(E)$. For $\sigma_{N N}(E)$ we use the fit to the available data in Ref. [27]. Using our height independent atmospheric composition, we simplify Eq. (15) as follows,

$$
\lambda_{N}(E, h)=\frac{\langle A\rangle}{\left\langle A^{2 / 3}\right\rangle} \frac{\mathrm{u}}{\sigma_{N N}(E)}=2.44 \frac{\mathrm{u}}{\sigma_{N N}(E)} .
$$

Here \langle\rangle denotes average and $\mathrm{u}$ is the atomic mass unit, that we write as

$$
\mathrm{u}=1660.54 \mathrm{mb} \mathrm{g} / \mathrm{cm}^{2} .
$$

We therefore find that in our approximations $\lambda_{N}(E)$ is independent of height.

*We recall that the elastic cross section contributes negligibly to the primary flux attenuation because the average elastic energy loss is very small, less than $1 \mathrm{GeV}$ at the high energies we consider. This can be seen using the differential elastic cross section $d \sigma_{e l} / d Q^{2}=\left(d \sigma_{e l} / d Q^{2}\right)_{Q^{2}=0} \exp \left(-b Q^{2}\right)$ with $b=\left[7.9+0.9 \ln p_{l a b}\right] \mathrm{GeV}^{-2}$, with $p_{l a b}$ in $\mathrm{GeV}[26$. Here $Q$ is the momentum transfer of the colliding proton of incoming momentum $p_{\text {lab }}$ and mass $M$. The mean energy loss is the mean value of $Q^{2} / 2 M$ (here $M$ is the target proton mass) namely $(1 / 2 M b)=67 \mathrm{MeV} /\left(1+0.1 \ln \left(p_{\text {lab }} / \mathrm{GeV}\right)\right)$. This is $46 \mathrm{MeV}$ at $E=100 \mathrm{GeV}$, and smaller at higher energies. 


\subsection{Charm production with MNR routines}

As we remarked before, the modified MNR routines are the first stage of our simulation. For a given energy $E$ of a primary incoming proton in the lab system, i.e. in the atmosphere reference frame, we generate a collision with a nuclear target at rest in the atmosphere, activating the MNR routines (primary event, $p N$ collision, with $N=$ $(p+n) / 2)$.

These routines generate total and differential cross sections through a VEGAS integration, which creates a large number of 'subevents', each one with a particular weight, which in the original MNR program are summed together to calculate the final cross sections.

It is easy to modify the program so that each of these subevents (together with its weight) can represent the production of a charm $c$ (or of a $c \bar{c}$ pair, or $c \bar{c}$ gluon, etc.) with given kinematics in any particular reference frame of interest. The original MNR routines can calculate single differential cross sections, in which the kinematics of only one final $c$ quark is available, and double differential cross sections, in which the full kinematics of the $c \bar{c}$ pair (plus an additional parton in NLO processes) becomes available, for each subprocess. We have used both these possibilities. We will refer to them as 'single' and 'double' modes. The 'single' is the mode we use to obtain all our results. We use the 'double' mode only to compare the results of the independent fragmentation model used in the evolution of cascades in the 'single' mode, with the more reliable string fragmentation model, which can only be used in the 'double' mode, as we explain below.

The MNR program [15, 16] contains all BORN and NLO processes. In the 'single' mode we can generate the following processes, with only the kinematics of the $c$ quark available,

$$
g g \rightarrow c X ; q \bar{q} \rightarrow c X(\mathrm{BORN}) \quad g g \rightarrow c X ; q \bar{q} \rightarrow c X ; q g \rightarrow c X(\mathrm{NLO})
$$

where q represents any light quark or antiquark. In the 'double' mode we have the following processes

$$
g g \rightarrow c \bar{c} ; \quad q \bar{q} \rightarrow c \bar{c}(\mathrm{BORN}) \quad g g \rightarrow c \bar{c} g ; \quad q \bar{q} \rightarrow c \bar{c} g ; \quad q g \rightarrow c \bar{c} q(\mathrm{NLO})
$$

for which the kinematics of all the outgoing partons is fully determined for each 'subevent'.

All the kinematical variables of the partons in the final state constitute the input for the next stage of the program, described in the next subsection.

An important characteristic of the first stage is that, besides $m_{c}, \mu_{R}$, and $\mu_{F}$, we can select any desired PDF to be used with the charm production routines. We have updated the set of PDFs in the original MNR program.

According to the discussion of Sect. 2, we use the MRS R1, MRS R2, CTEQ 3M and CTEQ 4M parton distribution functions, together with the values of $m_{c}, \mu_{R}$, and $\mu_{F}$ in Eqs. (10 四). 
As a concrete example of the integrals performed in our program, here we write the differential flux $\phi_{\mu}$ of muons (namely of $\mu^{+}+\mu^{-}$) with energy $E_{\mu}$ ( $\mu$ stands here for $\mu^{+}$or $\left.\mu^{-}\right)$in the 'single' mode $\left(\phi_{\mu}\right.$ has units $\left.\mathrm{cm}^{-2} \mathrm{~s}^{-1} \mathrm{sr}^{-1} \mathrm{GeV}^{-1}\right)$

$$
\begin{aligned}
\phi_{\mu}\left(E_{\mu}\right)= & \int_{E_{\mu}}^{\infty} d E \int_{0}^{\infty} d h \phi_{N}(E, X(h)) \sum_{A} n_{A}(h) \times \\
& \int_{E_{\mu}}^{E} d E_{c}\left[\frac{d \sigma\left(p A \rightarrow c Y ; E, E_{c}\right)}{d E_{c}}\right]_{M N R}\left[\frac{d N_{\mu}\left(c \rightarrow \mu ; E_{c}, E_{\mu}, h\right)}{d E_{\mu}}\right]_{\text {PYTHIA }} \\
+ & (c \rightarrow \bar{c}) .
\end{aligned}
$$

Here $n_{A}(h)$ is the number density of nuclei of atomic number $\mathrm{A}$ in the atmosphere, $E$ is the energy of the primary cosmic ray proton, $E_{c}$ the energy of the charm produced in the collision $p A \rightarrow c Y \quad(Y$ here stands for anything else). Using the relation $d \sigma(p A \rightarrow c Y) / d E_{c}=A d \sigma(p N \rightarrow c Y) / d E_{c}$, the sum over $A$ becomes $\sum_{A} n_{A}(h) A=$ $\rho(h) / u$. Using $d X=-\rho(h) d h$, Eq. (13), and normalizing to one the distribution in depth $X, \phi_{\mu}$ becomes

$$
\phi_{\mu}\left(E_{\mu}\right)=\int_{E_{\mu}}^{\infty} d E \int_{X_{0}}^{\infty} d X \phi_{N}(E, X=0) \frac{e^{-X / \Lambda_{N}(E)}}{\Lambda_{N}(E)}\left[\frac{f(h) \Lambda_{N}(E)}{u}\right],
$$

where, from Eqs.(14) and (16), $\Lambda_{N} / u=2.44\left[\sigma_{N N}\left(1-Z_{N N}\right)\right]^{-1}$ and

$$
f(h)=2 \int_{E_{\mu}}^{E} d E_{c}\left[\frac{d \sigma\left(p N \rightarrow c Y ; E, E_{c}\right)}{d E_{c}}\right]_{M N R}\left[\frac{d N_{\mu}\left(c \rightarrow \mu ; E_{c}, E_{\mu}, h\right)}{d E_{\mu}}\right]_{\text {PYTHIA }}
$$

Here the factor of 2 accounts for the muons produced by $\bar{c}$ (only c quarks are used in the program for simplicity); the $p N$ inclusive charm production cross section is computed with the MNR program (here are the integrations over the PDFs and partonic cross sections) and the last square bracket is the number of muons of energy $E_{\mu}$ which reach sea level, produced in the cascades simulated by PYTHIA. Each cascade is initiated by a $c$ quark (in the 'single' case) of energy $E_{c}$ and momentum $k$ (provided by the MNR routines) at a height $h$ chosen through a random number $R$ homogeneously distributed between 0 and 1, which gives the value of the $X$ probability distribution in Eq. (21), namely $R=e^{-X / \Lambda_{N}(E)}$.

\subsection{Cascade evolution with PYTHIA routines}

The parton $c$ (or partons in the 'double' case) generated by the first stage, namely by the MNR routines, are entered in the event list of PYTHIA and they become the starting point of the cascade generation.

PYTHIA first fragments the $c$ quark (in the 'single' mode, or all the partons in the 'double' mode) into hadrons, after showering, which can be optionally shut off. The charm quarks hadronize into $D^{0}, \bar{D}^{0}, D^{ \pm}, D_{s}^{ \pm}$and $\Lambda_{c}$. We used here the Peterson fragmentation function option. For each hadron produced, a simple routine added to PYTHIA decides if the hadron interacts in the atmosphere (loosing some energy) or 
decays. This is the same approach as in TIG. PYTHIA follows in this way the cascade in the atmosphere and populates the histograms of muons and neutrinos as a function of their different energies. We mention here a few important technical details. The 'single' and 'double' modes described before use different fragmentation models. In the 'single' mode only one $c$ quark is available and is entered at the beginning of the event list (with its energy and momentum in the partonic CM reference frame). In this case PYTHIA uses the 'independent fragmentation' model (see 25 for details). We only include $c$ quarks and at the end multiply the result by a factor of two to account for initial $\bar{c}$ quarks.

In the 'double' mode, instead, which we only use at the LO, we start with two $(c \bar{c})$ partons in the event list. In this case we opt to use the 'string fragmentation' model (Lund model, 25]). This model generally gives better results than the independent fragmentation, in which energy and momentum conservation have to be imposed a posteriori and whose results depend on the reference frame used, which empirically is chosen to be the partonic CM frame. To impose energy and momentum conservation in the independent fragmentation, we used the option $(\operatorname{MSTJ}(3)=1$, see again [25]) in which particles share momentum imbalance compensation according to their energy (roughly equivalent to boosting events to CM frame) but we have convinced ourselves that the results do not depend much on the way of imposing energy and/or momentum conservation, because trial runs with different options have given similar results for the fluxes.

Even if independent fragmentation is in general less desirable than string fragmentation, we use the 'single' mode as our main choice. The main reason to use the 'single' mode is that the simulations run in acceptably short times (a few days) on the SUN computers we use, while giving results practically identical to the 'double' mode in the comparisons we have made (see Fig. 6c). The simulation of the cascades in the 'double' mode takes between five and ten times longer. We tested the goodness of the independent fragmentation by comparing the outcome of fluxes computed at the Born level, in which the charm fluxes at production are identical (we put one $c$ in the atmosphere and multiply the outcome by two to account for the $\bar{c}$ in one case, and we put $c \bar{c}$ in the atmosphere, instead, in the second case) and the sole difference in both modes is due to the different fragmentation models used. The results were extremely close (at Born level the difference is less than $5 \%$, at energies above $10^{5} \mathrm{GeV}$ ), as can be seen in Fig. 6c.

Apart from the mentioned differences between the 'single' and 'double' modes, the simulations then proceed basically in the same way in both modes. For each of the 'subevents', i.e. for each set of initial parton(s) put in the event list, a certain height in the atmosphere is randomly chosen as explained above, this being the position at which the partons are generated from the initial proton-nucleon collision. This random height $h$ is generated in a way similar to TIG (see Ref. [11]), but different, because we include a correction for nucleon regeneration in nucleon-nucleon collisions by using $\Lambda_{N}$, the nuclear attenuation length, in Eq. (13) instead of $\lambda_{N}$, the interaction thickness (see Eqs. (14),(15) and (16)). The only difference compared to TIG (see Eq. (15) in 
the last paper of Ref. [11]) is the inclusion of the $\left(1-Z_{N N}\right)$ correction term. This was done because we could not include regenerated protons directly in our simulation of the cascades, since events and subevents are now created by the MNR routines and not by PYTHIA, as it was in TIG.

When parton showering is included at the beginning of the cascade simulation performed by PYTHIA, some double counting is present. The double counting appears when a LO diagram, for example $g g \rightarrow c \bar{c}$, with a subsequent splitting contained in PYTHIA, for example $c \rightarrow g c$ is summed to NLO diagram, $g g \rightarrow g c \bar{c}$ with the same topology, as if both diagram were independent, when actually the NLO contains the first contribution when the intermediate $c$ quark on mass shell. We have not tried to correct this double counting but have instead confronted the results obtained including showering (our standard option) with those excluding showering (in which case there is no double counting) and found very similar leptonic fluxes (see Fig. 6b).

The particles generated after the initial hadronization are then followed throughout the atmosphere and PYTHIA evolves the cascade with the same treatment of interactions and decays proposed by TIG. The final number of muons and neutrinos at sea level is therefore calculated considering all the 'subevents', each with its respective weight $W_{i}$ from the MNR program, which produce the final particles through all the possible decay channels of charmed particles decaying into prompt leptons. Since only the decay modes of charmed hadrons going into $\mu$ or $\nu_{\mu}$ or $\nu_{e}$ are left open in the simulation, and there are essentially just 2 modes for each charmed particle (for example: $D^{+} \rightarrow e^{+} \nu_{e}+$ anything , with branching ratio $=0.172 ; D^{+} \rightarrow \mu^{+} \nu_{\mu}+$ anything with branching ratio $=0.172$; all other channels closed), the branching ratios for each of these modes is fictitiously taken by PYTHIA to be $1 / 2$ and need to be normalized by multiplying by the actual branching ratio ( 0.172 for the example above) and dividing by $1 / 2$. Besides, since not all events are accepted by PYTHIA to generate a complete cascade, the result is normalized by dividing by the sum of all the weights of accepted events and multiplying it by the total c inclusive cross section.

\subsection{Summary}

To summarize, our computation of the final fluxes is organized as follows.

- An external loop over the primary energy $E$ generates an integration over $E$ in the range $10^{1}-10^{11} \mathrm{GeV}$.

- For each primary energy $E$, the MNR routines generate 'subevents' with weight $W_{i}$, for all the LO and NLO processes.

- Each subevent is assigned a random height (so that implicitly an integration over $h$ is performed) and all this is passed to PYTHIA as a definite set of parton(s) to be put at the beginning of the event list.

- For each of these 'subevents', PYTHIA treats showering (in our standard option), hadronization and evolution of the cascade in the atmosphere, and generates the final leptons. 
- For each decay channel of interest, the produced leptons are weighted with $W_{i}$ and then summed into the final fluxes.

\section{$4 \quad$ Neutrino and muon fluxes}

Figs. 4-6 show the results of our simulations. Fig. 4 shows the total inclusive charmanticharm production cross sections $\sigma_{c \bar{c}}$, and the $K$ factor for $c$ production, namely the ratio between the NLO and Born cross sections, $K_{c}=\sigma_{c \bar{c}}^{N L O} / \sigma_{c \bar{c}}^{B o r n}$, for the four PDFs we consider and for TIG. Fig. 5 shows our main results obtained with our default choice of options: a 'single' mode calculation including the contributions from all processes in Eq. (18) and with parton showering included in the cascade simulation performed by PYTHIA). Finally Fig. 6 shows the relative importance of the processes included in the fluxes and a comparison of the 'single' and 'double' modes and of the 'on' and 'off' showering options.

In Fig. 4a, the total inclusive charm-anticharm production cross sections $\sigma_{c \bar{c}}$ are plotted over the energy range needed by our program, $E \leq 10^{11} \mathrm{GeV}$, for our four different PDFs. They were calculated using the MNR program, with the 'calibration' described in Sect. 2, up to the NLO contribution. For comparison, we also show the cross section used by TIG and the Born (LO) contribution for one of the PDFs, MRS R1. We see in the figure that all our cross sections agree at low energies, as expected due to our 'calibration' at $250 \mathrm{GeV}$, and are very similar for energies up to $10^{6}-10^{7}$ $\mathrm{GeV}$. At higher energies they diverge, differing by at most $50 \%$ at the highest energy we use, $10^{11} \mathrm{GeV}$. In fact, at energies beyond $10^{7} \mathrm{GeV}$, the CTEQ 3M cross section becomes progressively larger than the CTEQ 4M and MRS R2 cross sections, which are very close to each other. The MRS R1 becomes on the contrary progressively lower than the other three.

We see in Fig. 4a that for energies above $10^{4} \mathrm{GeV}$ our cross sections are considerably higher than the one used by TIG. This difference can be traced in part to the use by TIG of an option of PYTHIA by which the gluon PDF is extrapolated to $x \leq 10^{-4}$ with $\lambda=0.08$, while all the PDFs we use have a higher value of $\lambda \simeq 0.2-0.3$. And in part to TIG scaling the LO cross sections obtained with PYTHIA by a constant $K$ factor of 2 , while at large energies the $K$ factor is actually larger than 2 by about $10-15 \%$ (see Fig. 4b).

In Fig. 4b we explicitly show the $K$ factor for $c$ production, namely the ratio between the NLO and Born cross sections, $K_{c}=\sigma_{c \bar{c}}^{N L O} / \sigma_{c \bar{c}}^{B o r n}$, for our PDFs and for TIG. All the $K_{c}$ values are around the usually cited value of 2 for most of the intermediate energies, but are larger at the lowest energies and also at the highest energies (except for CTEQ 3M), and they all are within about $15 \%$ of each other.

Fig. 5 contains three sets of figures, one for each lepton: $\mu, \nu_{\mu}$ and $\nu_{e}$. The left figure of each set shows the $E^{3}$-weighted vertical prompt fluxes, for all our PDFs up to NLO (labelled 'NLO') and, as an example, the LO (labelled 'BORN') for MRS R1, together

with the total fluxes up to NLO of TIG, both from prompt and conventional sources 
(dotted lines). The right part of each set shows the corresponding $K_{l}$ value (where $\left.l=\mu, \nu_{\mu}, \nu_{e}\right)$, i.e. the ratio of the total NLO flux to the Born flux of the figure on the left. The figures show that our fluxes are higher than those of TIG for $E>10^{3} \mathrm{GeV}$. Leaving apart differences in the two simulations that cannot be easily quantified, this discrepancy can largely be explained by the different cross sections used by TIG and us: the TIG cross section is lower than ours for $E>10^{4} \mathrm{GeV}$. Using a value of $\lambda$ similar to TIG $(\lambda \simeq 0)$ at small $x$, we obtain fluxes similar to those of TIG at energies above $10^{6} \mathrm{GeV}$ [24].

In particular, our fluxes are all larger than TIG by factors of 3 to 10 at the highest energies, what puts our fluxes in the bulk-part of previous estimates (see Refs. 12 ,

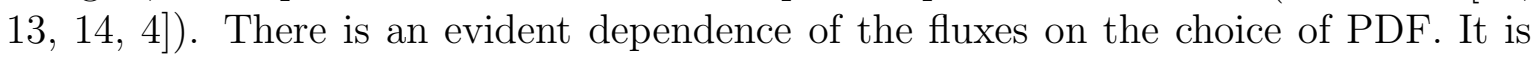
remarkable that MRS R2 and CTEQ 4M give very similar results. Those of the MRS $\mathrm{R} 1$ become lower and those of the older CTEQ 3M PDF become higher as the energy increases (both differing by about 30-50\% at the highest energies with respect to the MRS R2-CTEQ 4M fluxes). This is due to the intrinsic differences of the PDF packages used and the consequent different extrapolated values of $\lambda$ at small $x$ or high energies.

The CTEQ 3M fluxes were included to compare our results with those of Ref. [17]. We find our CTEQ 3M results to be close to those of Ref. [17], in spite of the very different approaches used in the two calculations. Our fluxes lie between the two curves for CTEQ 3M shown in Fig.8 of Ref. [17], corresponding to different choices of renormalization and factorization scales. Our fluxes are lower (by 30-40\% at $10^{7} \mathrm{GeV}$ ), than the main CTEQ 3M choice of Ref. [17] (solid line of their Fig.8), which is calculated using values of $\mu_{R}, \mu_{F}$ and $m_{c}$ similar to ours. Our cross section for charm production, for the CTEQ 3M case, is essentially equal to the one used in Ref. [17] (shown in their Fig. 2), so the discrepancies in the final fluxes are to be explained in terms of the differences in the cascade treatment. It is very difficult to trace the reasons for these differences.

We also see in the figures that, for each PDF, the fluxes for the different leptons are very similar: those for $\nu_{\mu}$ neutrinos and $\nu_{e}$ are essentially the same, those for muons are only slightly lower (about $10 \%$ less at the energies of interest). Also the $K_{l}$ 's don't differ much for the three leptons, apart from some unphysical fluctuations especially evident at the highest energies. Even if they differ the PDF, they all show a similar energy dependence, namely they increase at low energies and sometimes at high energies also. This behavior is also similar to that of the $K_{c}$ factors in Fig. 4b, but with a weaker overall energy dependence, as expected, since the leptons of a given energy result from $c$ quarks with a range of higher energies.

The $K_{l}$ factors are all within the range $2.1-2.5$ : they are approximately 2.2 for MRS R1, 2.4 for MRS R2 and CTEQ 4M, and 2.3 for CTEQ 3M. Thus, our analysis shows that evaluating the lepton fluxes only at the Born level, and multiplying them by an overall $K_{l}$ factor of about $2.2-2.4$ (i.e. 10 to $20 \%$ larger than the value of 2 used by TIGI), can be good enough to evaluate the NLO fluxes within about $10 \%$. Thus we find the approach used by TIG, who multiplied the LO fluxes obtained with

\footnotetext{
${ }^{\dagger}$ We note that in the original TIG model there is no distinction between $K_{c}$ and $K_{l}$ factors since
} 
PYTHIA by two, essentially correct, except for their relatively low $K$ factor and the discrepancies existing even at Born level between our fluxes and those of TIG. In fact, as we mentioned previously, the differences between our final results and those of TIG depend mostly on the different total inclusive $c$ cross sections, which can be traced to the extrapolation of the gluon PDF at small $x$ rather than to the $\mathrm{K}$ factor. Possible causes of the different results due to the intrinsic differences of the computer simulations cannot be easily quantified.

In Fig. 6 we address three issues. First, we show that the fluxes can be obtained within about $30 \%$ with just the gluon-gluon process. This would speed up the simulations and, when using the MNR program, would give (contrary to intuition) higher fluxes than those actually derived from all processes. Secondly, we show that the fluxes obtained including or excluding showering in the simulation made by PYTHIA (we included showering in our standard options) do not differ significantly. The third issue we deal with is the difference between the 'single' and 'double' modes described in Sect. 3. We show that at LO the results from a 'double' mode calculation coincide with those of the much shorter 'single' mode, that we use in all our calculations. Let us deal with these three issues in turn.

In Fig. 6a we show, for a given PDF, the MRS R1, the relative importance of the different processes contributing to the final fluxes. The solid line is the total flux obtained as the sum of all the processes of Eq. (18) and the dotted line shows the result of only gluon-gluon fusion $(g g)$, the sum of Born $(g g)$ and pure NLO (excluding Born) $g g$ processes. Also shown are the separate contributions only at the Born and at the NLO (excluding LO) of both $g g$ and quark-antiquark $(q \bar{q})$ fusion, what clearly shows that $g g$ dominates. This is to be expected because the gluon PDF is either much larger than (for $x<0.1$ ) or comparable to (for $x \simeq \mathcal{O}(0.1)$ ) the quark PDFs. The figure plots the absolute value of the quark-gluon $(q g)$ terms because, for the values of the factorization scale that we employ in our calculations, these terms are negative. This is due to the way the original MNR calculation is subdivided into processes. In fact, in the MNR program, a part of the quark-gluon contribution to the cross sections is already contained in other processes, and must be subtracted in the processes labelled as $q g$. The amount subtracted depends on the factorization scale $\mu_{F}$ and may drive the $q g$ contribution negative. Roughly speaking, if $\mu_{F}$ is small the $q g$ term is positive, otherwise (as in our case) the term is negative. The absolute value of the $q g$ term is in between the $q \bar{q}$ and the $g g$ terms, what makes negative the sum of all the processes different from $g g$. Thus, gluon-gluon processes alone give a result slightly larger than the total, by about $30 \%$.

In Fig. 6b we check the effect of shutting off the showering option available in PYTHIA. We study only one specific case, the MRS R1. The overall effect is minimal: the exclusion of showering slightly increases the energy of the parent charmed hadrons and therefore causes the final fluxes of lepton daughters to move towards higher energies; the effect is barely noticeable and just slightly more important for the Born

only the Born level is considered. Their $K=2$ factor is just a multiplicative constant which can be considered either a $K_{c}$ or a $K_{l}$. 
fluxes (the overall difference is about 5\%). When showering is included some double counting occurs, whose effect must be smaller than the difference between the results with showering on and off (since in this case no double counting occurs).

Finally in Fig. 6c we confront the 'single' and 'double' modes of the program, for just one PDF, MRS R1, at Born level. At this level, the calculation of the charm flux at production is identical (we obtain the fluxes from $c$ and multiply by two at the end to account for the $\bar{c}$ in one case, and we obtain the fluxes directly from $c \bar{c}$ in the other). So, what is actually compared in the two modes at the Born level is the fragmentation model: independent fragmentation in the 'single' mode and string (Lund) fragmentation in the 'double' mode. The results from both modes at the Born level are almost identical: as already remarked the difference is less than $5 \%$ for energies above $10^{6} \mathrm{GeV}$.

\section{Conclusions}

We have used the actual next-to-leading order perturbative QCD calculations of charm production cross sections, together with a full simulation of the atmospheric cascades, to obtain the vertical prompt fluxes of neutrinos and muons.

Our treatment is similar to the one used by TIG, except for the very important difference of including the true NLO contribution, while TIG used the LO charm production cross section multiplied by a constant $K$ factor of 2 to bring it in line with the next-to-leading order values. The main goal of this paper is to examine the validity of TIG's procedure by computing the ratio of the fluxes obtained with the NLO charm production cross section versus those obtained with the LO cross section.

These ratios, the $K_{l}$ factors are between 2.1 and 2.5 for the different gluon PDFs in the energy range from $10^{2}$ to $10^{9} \mathrm{GeV}$ (see Fig. 5). Consequently, our analysis shows that evaluating the lepton fluxes only at the Born level, and multiplying them by an overall factor of about $2.2-2.4$, slightly dependent on the PDF, can be good enough to evaluate the NLO fluxes within about 10\%. Therefore, we find the approach used by TIG (i.e. multiplying the LO fluxes by two) essentially correct, except for their relatively low $K$ factor. We find different lepton fluxes than TIG, but this is mostly due to the discrepancies, even at Born level, between our charm production cross sections and TIG's.

In fact, the prompt neutrino and muon fluxes found by TIG were lower than the lowest previous prediction. We find here instead fluxes in the bulk part of those previous

predictions. This difference can be traced largely to the use by TIG of an option of PYTHIA by which the gluon PDF is extrapolated for $x \leq 10^{-4}$ with $\lambda=0.08$, while all the PDFs in this paper have a higher value of $\lambda \simeq 0.2-0.3$. Using a value of $\lambda$ similar to TIG $(\lambda \simeq 0)$ we obtain fluxes similar to those of TIG, at energies above $10^{6}$ $\mathrm{GeV}[24$. 


\section{Acknowledgements}

The authors would like to thank the Aspen Center For Physics, where this work was initiated, for hospitality, and M. Mangano and P. Nason for the MNR program and helpful discussions. This research was supported in part by the US Department of Energy under grant DE-FG03-91ER40662 Task C. 


\section{References}

[1] T.K. Gaisser, "Cosmic Rays and Particle Physics" (Cambridge University Press, 1990).

[2] A. Kernan and G. Van Dalen, Phys. Rep. 106 (1984) 297.

S.P.K. Tavernier, Rep. Progr. Phys. 50 (1987) 1439.

[3] H. Inazawa and K. Kobayakawa, Progr. Theor. Phys. 69 (1983) 1195.

[4] E. Zas, F. Halzen, and R.A. Vázquez, Astroparticle Phys. 1 (1993) 297.

[5] E.V. Bugaev et al., in 3rd NESTOR International Workshop Pylos (Greece, 1993), Resvanis L.K. (ed.), p. 268.

[6] J.A. Appel, Annu. Rev. Nucl. Part. Sci. 42 (1992) 367.

S. Frixione, M.L. Mangano, P. Nason, and G. Ridolfi, Nucl. Phys. B431 (1994) 453.

P.E. Karchin, LISHEP95 (Rio de Janeiro, 1995), FERMILAB-Conf-95.053-E. L. Rossi, Nucl. Phys. B (Proc. Suppl.) 50 (1996) 172.

[7] E769 Collaboration, G.A. Alves et al., Phys. Rev. Lett. 77 (1996) 2388.

[8] E769 Collaboration, G.A. Alves et al., Phys. Rev. Lett. 77 (1996) 2392.

[9] WA92 Collaboration, M.I. Adamovich et al., Nucl. Phys. B495 (1997) 3.

[10] S. Frixione, M.L. Mangano, P. Nason, and G. Ridolfi, preprint CERN-TH/97-16, hep-ph/9702287.

[11] M. Thunman, G. Ingelman, and P. Gondolo, Nucl. Phys. B (Proc. Suppl.) 43 (1995) 274.

P. Gondolo, G. Ingelman, and M. Thunman, Nucl. Phys. B (Proc. Suppl.) 48 (1996) 472.

M. Thunman, Ph.D. Thesis, Uppsala University, 1996.

M. Thunman, G. Ingelman, and P. Gondolo, Astroparticle Phys. 5 (1996) 309.

[12] E.V. Bugaev, V.A. Naumov, S.I. Sinegovsky, and E.S. Zaslavskaya, Nuovo Cimento C12 (1989) 41.

[13] C. Castagnoli et al., Nuovo Cimento A82 (1984) 78.

[14] M. C. Gonzalez-Garcia, F. Halzen, R.A. Vázquez and E. Zas, Phys. Rev. D49 2310 .

[15] M.L. Mangano, P. Nason, and G. Ridolfi, Nucl. Phys. B373 (1992) 295; Nucl. Phys. B405 (1993) 507. 
[16] P. Nason, S. Dawson, and R.K. Ellis, Nucl. Phys. B303 (1988) 607.

P. Nason, S. Dawson, and R.K. Ellis, Nucl. Phys. B327 (1989) 49; Erratum B355 (1990) 260.

[17] L. Pasquali, M.H. Reno, and I. Sarcevic, preprint hep-ph/9806428.

[18] A.D. Martin, R.G. Roberts and W.J. Stirling, Phys. Lett. 387 (1996) 419.

[19] H.L. Lai et al., Phys. Rev. D51 (1995) 4763.

[20] H.L. Lai et al., Phys. Rev. D55 (1997) 1280.

[21] P. Harriman et al., Phys. Rev. D42 (1990) 798.

P. Sutton et al., Phys. Rev. D45 (1992) 2349.

[22] J. Kwiecinski, A.D. Martin, and P.J. Sutton, Phys. Lett. B264 (1991) 199. J. Kwiecinski, A.D. Martin, and P.J. Sutton, Phys. Rev. D44 (1991) 2640.

A.D. Martin, Acta Physica Pol. B25 (1994) 265.

J. Kwiecinski, Phys. Rev. D52 (1995) 1445.

[23] E.A. Kuraev, L.N. Lipatov and V.S. Fadin, Sov. Phys. JETP 45 (1977) 199.

Ya. Ya. Balitsky, L.N. Lipatov, Sov. J. Nucl. Phys. 28 (1978) 822.

[24] G. Gelmini, P. Gondolo, and G. Varieschi, in preparation.

[25] T. Sjöstrand, Computer Phys. Commun. 82 (1994) 74.

[26] K. Goulianos, Phys. Rep. 101 (1983) 169.

[27] Review of Particle Physics, Phys. Rev. D54 (1996) 1. 


\section{FIGURE CAPTIONS}

Fig. 1 Comparison of experimental data for $\sigma_{c \bar{c}}$ with MNR predictions for different $m_{c}$ values: (a) in $p N$ collisions ([10], Table [), (b) in $\pi N$ collisions ([10], Table [2) (PDF: MRS R1).

Fig. 2 Comparison of differential cross sections for $\left(D^{+}, D^{-}, D^{0}, \bar{D}^{0}, D_{S}^{+}\right.$and $\left.D_{S}^{-}\right)$production, calculated using MNR at different $m_{c}$ values, with E769 data for $p N$ and $\pi N[\mathbb{8}]:$ (a) $d \sigma / d x_{F}$, (b) $d \sigma / d p_{T}^{2}\left(x_{F}>0\right)$ (PDF: MRS R1).

Fig. 3 Comparison of differential cross sections for $\left(D^{+}, D^{-}, D^{0}, \bar{D}^{0}\right)$ production, calculated using MNR at different $m_{c}$ values, with WA92 data for $\pi N$ [9]: (a) $d \sigma / d x_{F}$, (b) $d \sigma / d p_{T}^{2}\left(x_{F}>0\right)$ (PDF: MRS R1).

Fig. 4 (a) Total cross sections for charm production $\sigma_{c \bar{c}}$ up to NLO, for different PDFs, compared to the one used in the TIG model [11] (for MRS R1 we also show the Born cross section). (b) Related $K_{c}$ factors.

Fig. $5 E^{3}$-weighted vertical prompt fluxes, for different PDFs, at NLO (for MRS R1 we also show the Born flux), for the three types of leptons considered, compared to the TIG [11] conventional and prompt fluxes (left figures) and the related $K_{l}$ factors for each case (right figures).

Fig. 6 (a) Contributions of the different Born and NLO processes to the total $E^{3}$ weighted vertical prompt fluxes. (b) Comparison of the fluxes with or without the showering option, at Born and NLO. (c) Comparison of the fluxes calculated in the 'single' or 'double' mode, at Born only (PDF: MRS R1). 


\begin{tabular}{|c|c|c|c|c|c|}
\hline & $\begin{array}{c}\text { Beam } \\
\text { Energy } \\
(\mathrm{GeV})\end{array}$ & $\begin{array}{c}\sigma_{+}\left(x_{F}>0\right) \\
(\mu b)\end{array}$ & $\begin{array}{c}\sigma_{c \bar{c}}(\mathrm{EXP} .) \\
(\mu b)\end{array}$ & $\begin{array}{c}\sigma_{c \bar{c}}(\mathrm{MNR}) \\
(\mu b)\end{array}$ & PDF \\
\hline $\begin{array}{l}p N \\
\mathrm{E} 769 \text { [7] }\end{array}$ & 250 & $\begin{array}{c}\sigma_{+}\left(D^{+}, D^{-}\right)=3.3 \pm 0.4 \pm 0.3 \\
\sigma_{+}\left(D^{0}, \bar{D}^{0}\right)=5.7 \pm 1.3 \pm 0.5\end{array}$ & $13.5 \pm 2.2$ & $\begin{aligned} & 13.54 \\
m_{c}= & 1.185 \mathrm{GeV}\end{aligned}$ & MRS R1 \\
\hline $\begin{array}{ll}p N \\
\mathrm{E} 769 \text { 迎 }\end{array}$ & 250 & $\begin{array}{c}\sigma_{+}\left(D^{+}, D^{-}\right)=3.3 \pm 0.4 \pm 0.3 \\
\sigma_{+}\left(D^{0}, \bar{D}^{0}\right)=5.7 \pm 1.3 \pm 0.5\end{array}$ & $13.5 \pm 2.2$ & $\begin{array}{c}13.43 \\
m_{c}=1.31 \mathrm{GeV}\end{array}$ & MRS R2 \\
\hline 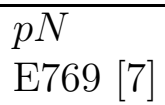 & 250 & $\begin{array}{c}\sigma_{+}\left(D^{+}, D^{-}\right)=3.3 \pm 0.4 \pm 0.3 \\
\sigma_{+}\left(D^{0}, \bar{D}^{0}\right)=5.7 \pm 1.3 \pm 0.5\end{array}$ & $13.5 \pm 2.2$ & $\begin{array}{c}13.59 \\
m_{c}=1.27 \mathrm{GeV}\end{array}$ & CTEQ4M \\
\hline $\begin{array}{l}p N \\
\mathrm{E} 769 \text { [7] }\end{array}$ & 250 & $\begin{array}{c}\sigma_{+}\left(D^{+}, D^{-}\right)=3.3 \pm 0.4 \pm 0.3 \\
\sigma_{+}\left(D^{0}, \bar{D}^{0}\right)=5.7 \pm 1.3 \pm 0.5\end{array}$ & $13.5 \pm 2.2$ & $\begin{array}{c}13.45 \\
m_{c}=1.24 \mathrm{GeV}\end{array}$ & CTEQ3M \\
\hline
\end{tabular}

Table 1: Data on total cross sections for charm production for $p N$ collisions, from E769 experiment, have been converted to $c \bar{c}$ cross sections and compared to the predictions of the MNR program running at slightly different values of the charm mass $\mathrm{m}_{c}$, using different PDFs.

\begin{tabular}{|c|c|c|c|c|c|}
\hline & $\begin{array}{l}\text { Beam } \\
\text { Energy } \\
(\mathrm{GeV})\end{array}$ & $\begin{array}{c}\sigma_{+}\left(x_{F}>0\right) \\
(\mu b)\end{array}$ & $\begin{array}{c}\sigma_{c \bar{c}}(\mathrm{EXP} .) \\
(\mu b)\end{array}$ & $\begin{array}{c}\sigma_{c \bar{c}}(\mathrm{MNR}) \\
(\mu b) \\
m_{c}=1.185 \mathrm{GeV}\end{array}$ & $\begin{array}{c}\sigma_{c \bar{c}}(\mathrm{MNR}) \\
(\mu b) \\
m_{c}=1.250 \mathrm{GeV}\end{array}$ \\
\hline $\begin{array}{l}\pi^{-} N \\
\text { E769 [7] }\end{array}$ & 210 & $\begin{array}{l}D^{+}, D^{-}: 1.7 \pm 0.3 \pm 0.1 \\
D^{0}, \bar{D}^{0}: 6.4 \pm 0.9 \pm 0.3\end{array}$ & $9.7 \pm 1.2$ & 14.08 & 10.64 \\
\hline $\begin{array}{l}\pi^{-} N \\
\text { E769 [7] }\end{array}$ & 250 & $\begin{array}{l}D^{+}, D^{-}: 3.6 \pm 0.2 \pm 0.2 \\
D^{0}, \bar{D}^{0}: 8.2 \pm 0.7 \pm 0.5\end{array}$ & $14.2 \pm 1.1$ & 16.54 & 12.56 \\
\hline $\begin{array}{l}\pi^{+} N \\
\text { E769 [7] }\end{array}$ & 250 & $\begin{array}{l}D^{+}, D^{-}: 2.6 \pm 0.3 \pm 0.2 \\
D^{0}, \bar{D}^{0}: 5.7 \pm 0.8 \pm 0.4\end{array}$ & $10.0 \pm 1.2$ & 16.54 & 12.56 \\
\hline $\begin{array}{l}\pi^{ \pm} N \\
\text { E769 [7] } \\
\end{array}$ & 250 & $\begin{array}{l}D^{+}, D^{-}: 3.2 \pm 0.2 \pm 0.2 \\
D^{0}, \bar{D}^{0}: 7.2 \pm 0.5 \pm 0.4 \\
\end{array}$ & $12.5 \pm 0.8$ & 16.54 & 12.56 \\
\hline $\begin{array}{l}\pi^{-} N \\
\text { WA92 [9] }\end{array}$ & 350 & $\begin{array}{l}D^{+}, D^{-}: 3.28 \pm 0.08 \pm 0.29 \\
D^{0}, \bar{D}^{0}: 7.78 \pm 0.14 \pm 0.52\end{array}$ & $13.3 \pm 0.7$ & 22.22 & $\begin{array}{c}17.06 \\
\left(13.5 \text { for } m_{c}\right. \\
=1.31 \mathrm{GeV})\end{array}$ \\
\hline
\end{tabular}

Table 2: Data on total cross sections for charm production for $\pi N$ collisions, from E769 and WA92 experiments, have been converted to $c \bar{c}$ cross sections and compared to the predictions of the MNR program running at slightly different values of the charm mass $\mathrm{m}_{c}$, using MRS R1. 

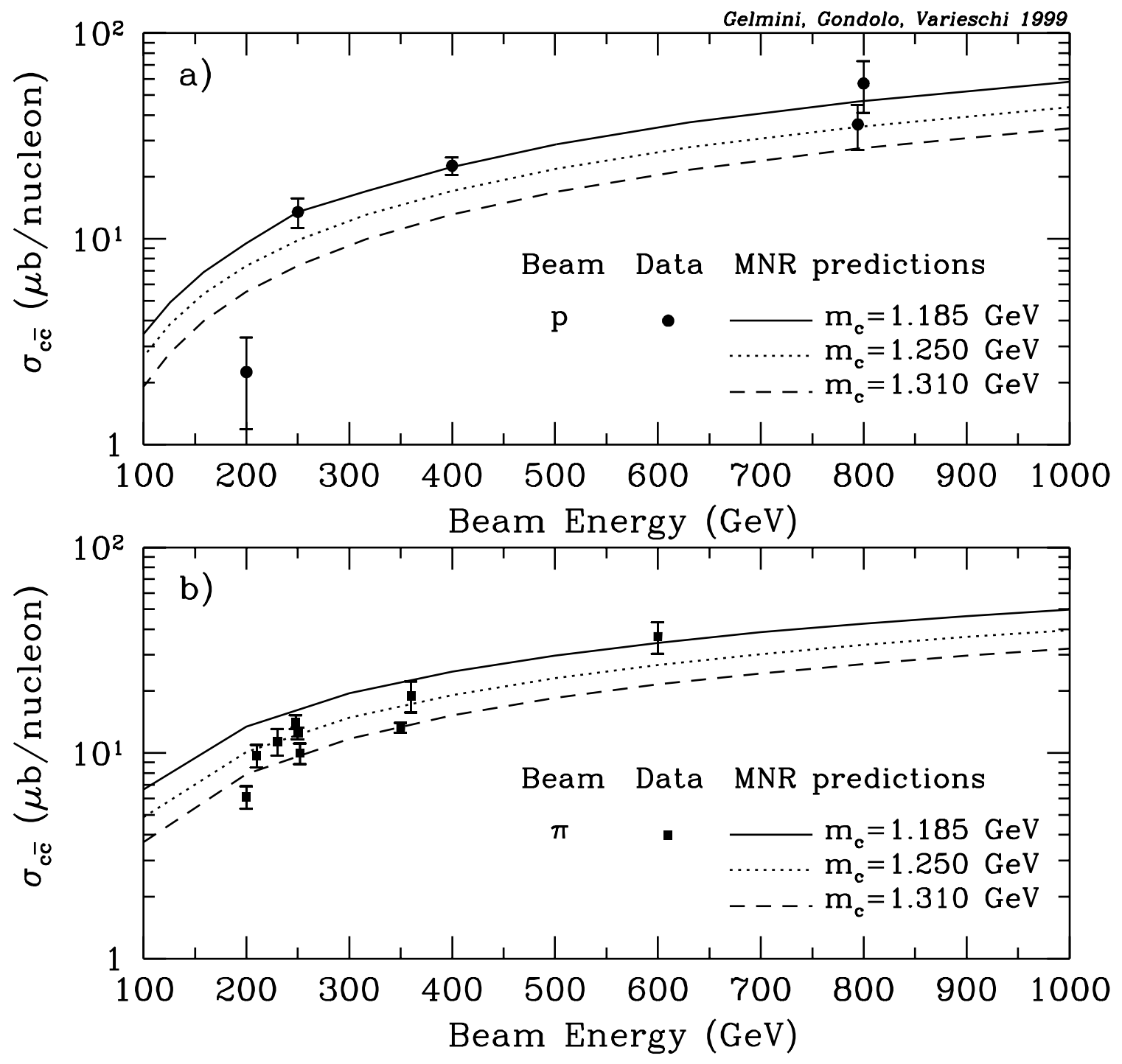

Figure 1: 


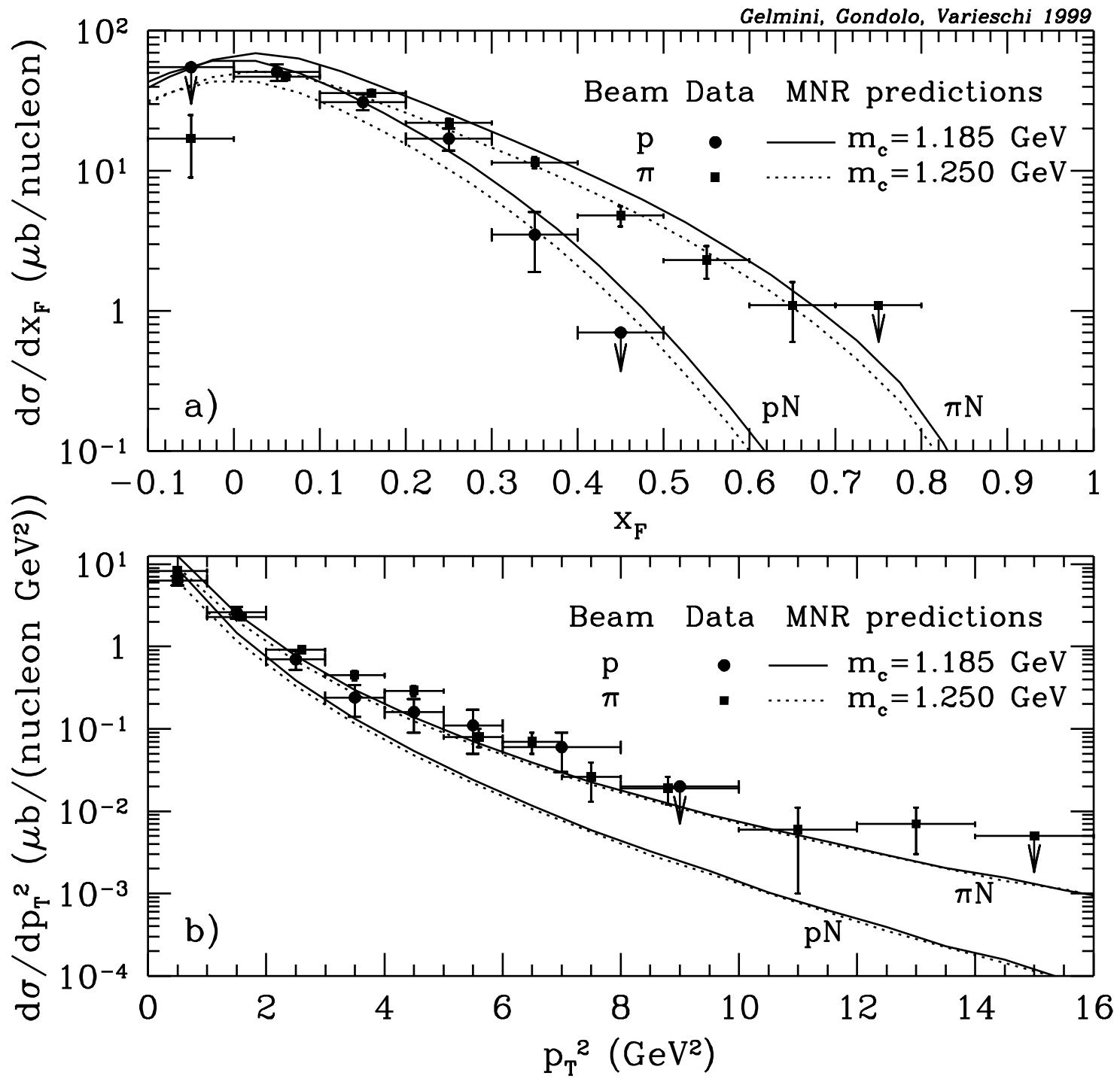

Figure 2: 


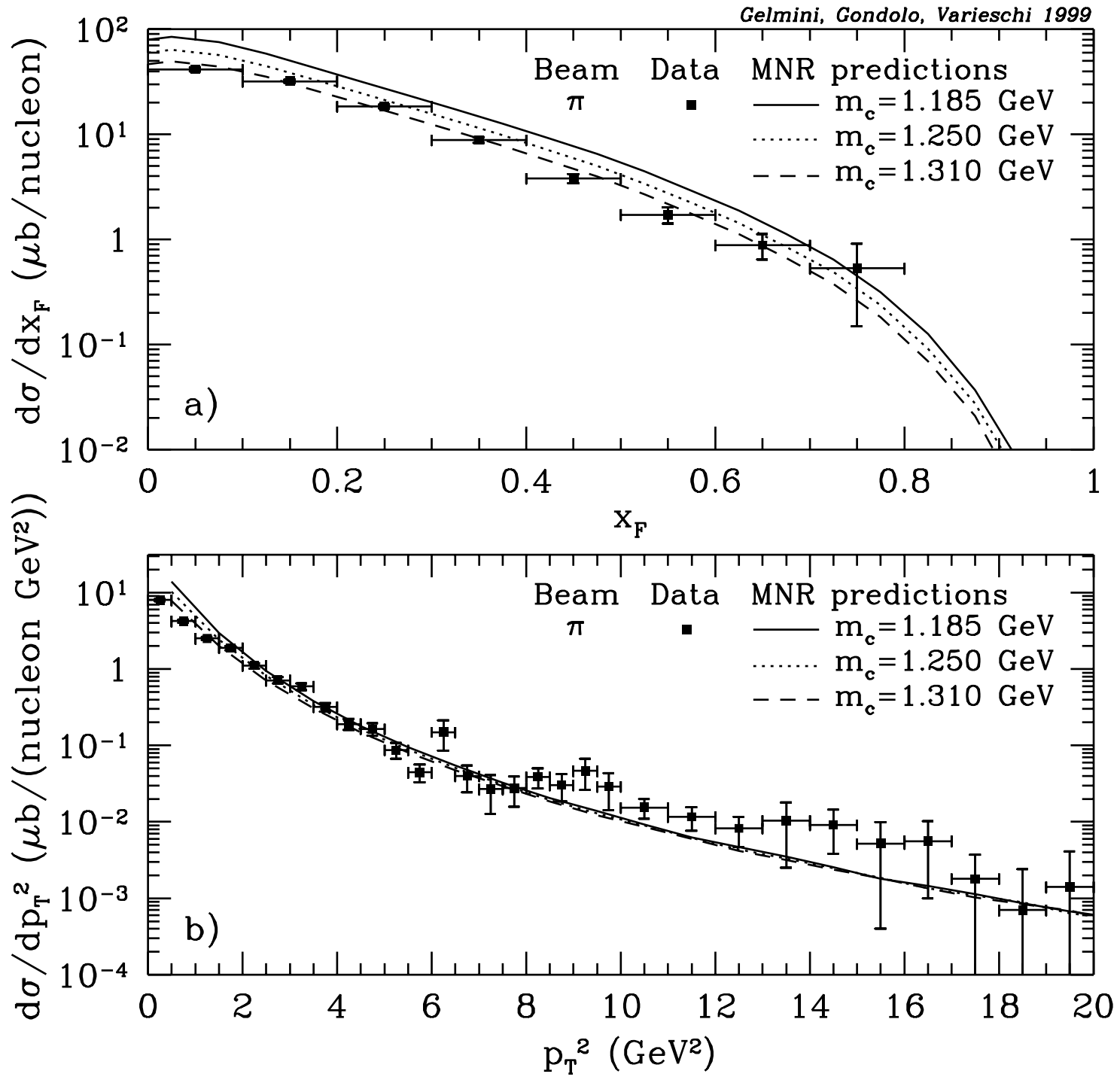

Figure 3: 

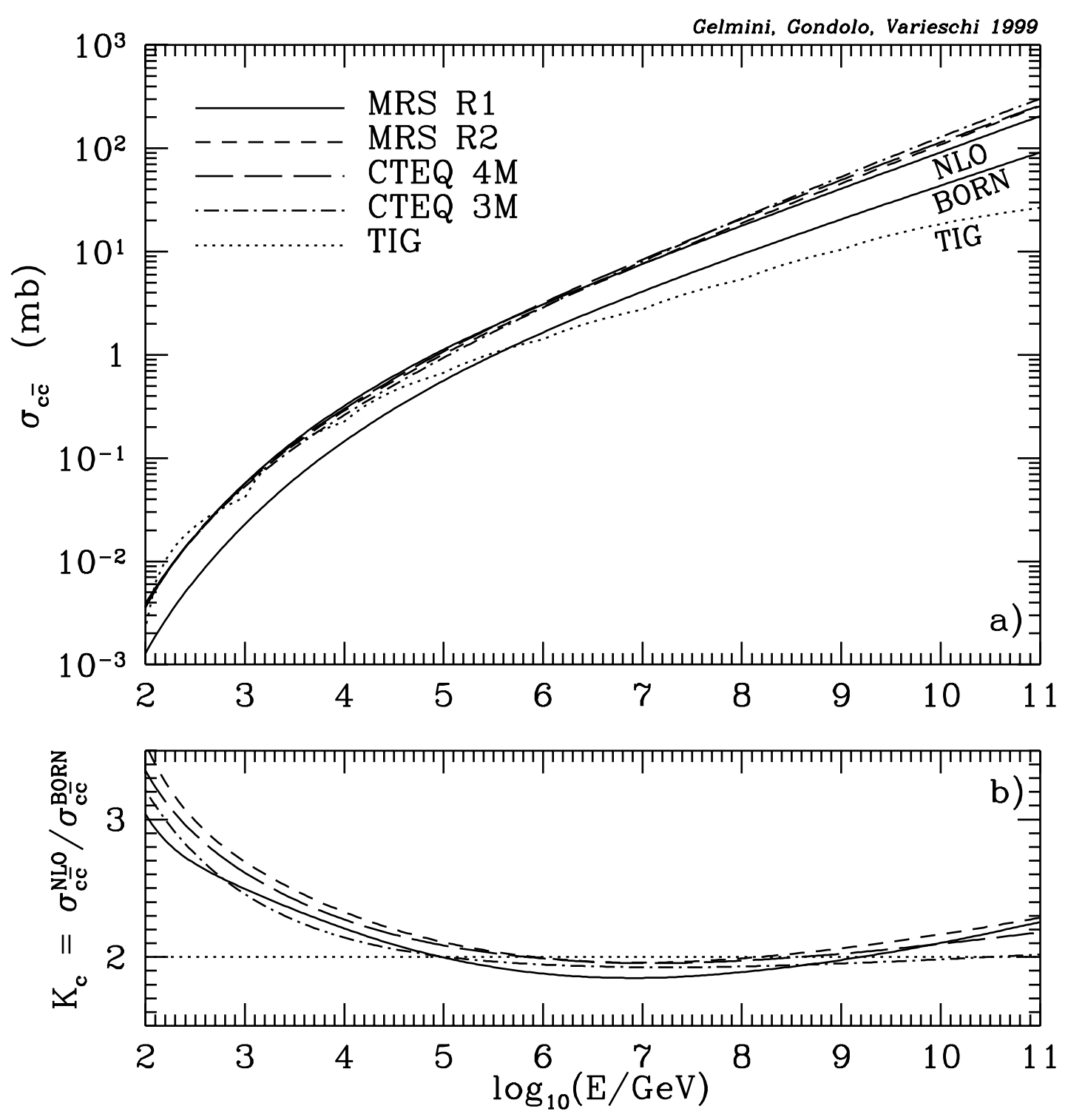

Figure 4: 

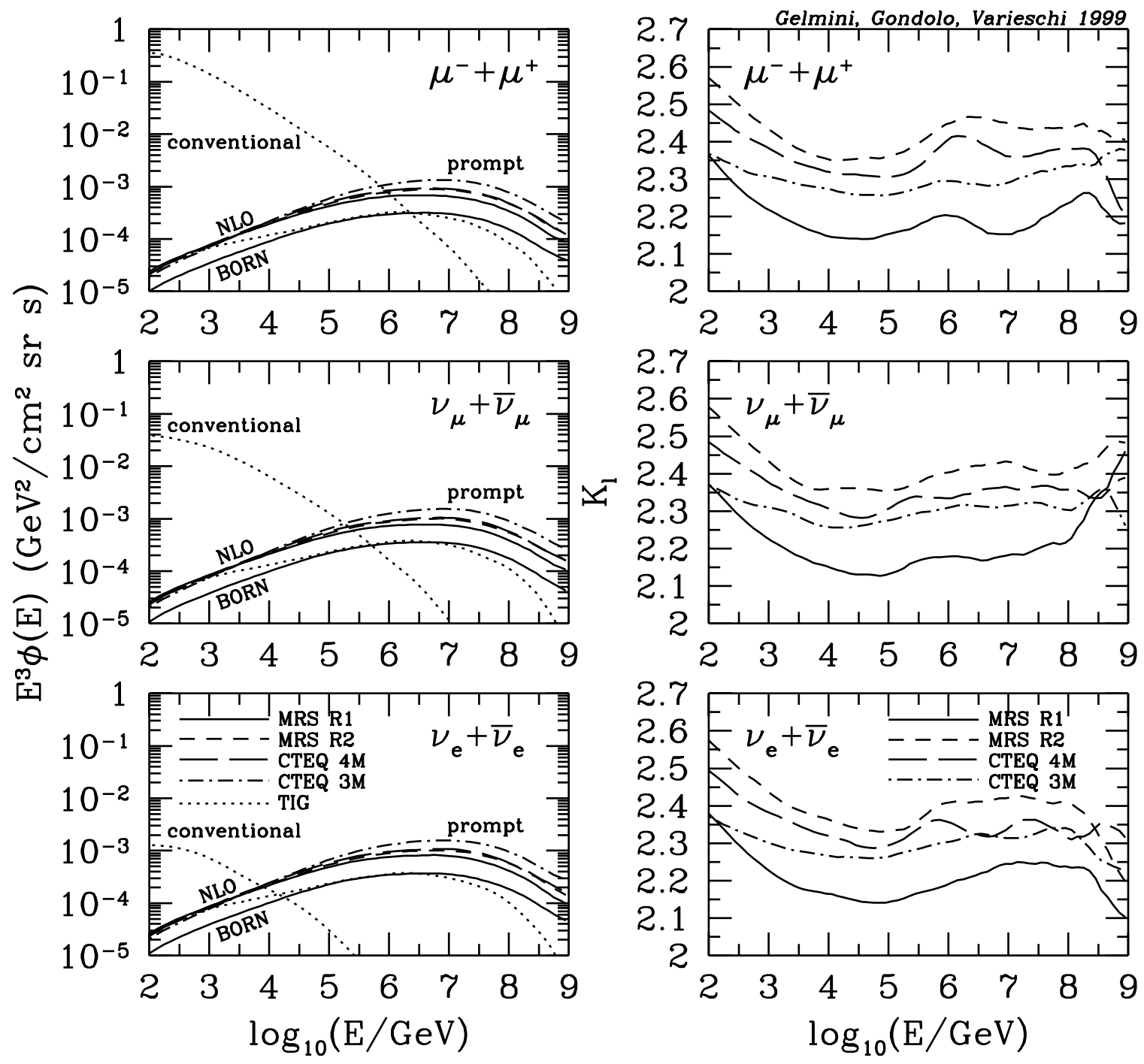

Figure 5: 


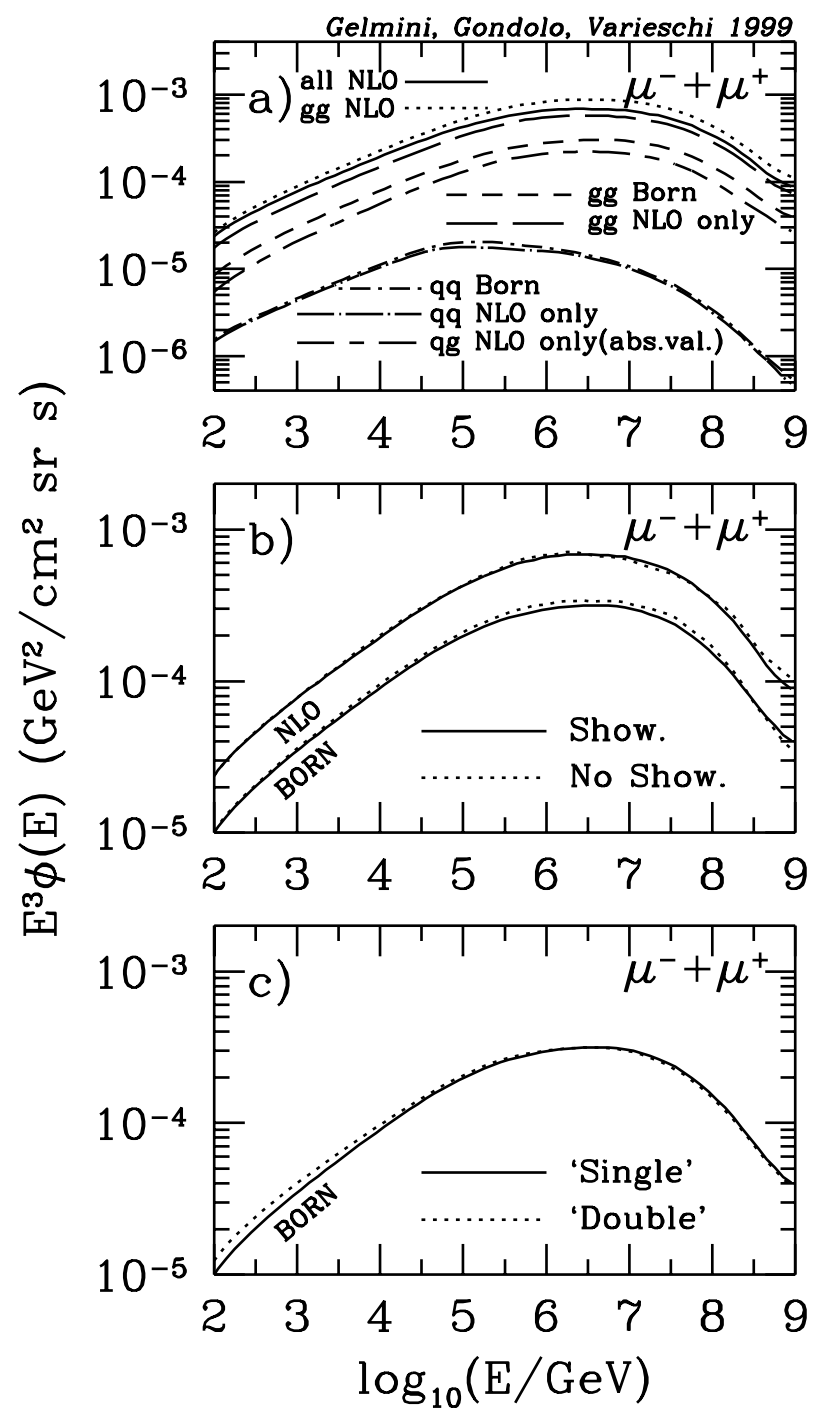

Figure 6: 